ARTICLE

\title{
Laser nano-filament explosion for enabling open-grating sensing in optical fibre
}

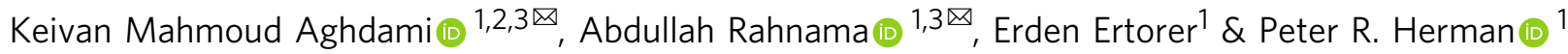

Embedding strong photonic stopbands into traditional optical fibre that can directly access and sense the outside environment is challenging, relying on tedious nano-processing steps that result in fragile thinned fibre. Ultrashort-pulsed laser filaments have recently provided a non-contact means of opening high-aspect ratio nano-holes inside of bulk transparent glasses. This method has been extended here to optical fibre, resulting in high density arrays of laser filamented holes penetrating transversely through the silica cladding and guiding core to provide high refractive index contrast Bragg gratings in the telecommunication band. The point-by-point fabrication was combined with post-chemical etching to engineer strong photonic stopbands directly inside of the compact and flexible fibre. Fibre Bragg gratings with sharply resolved $\pi$-shifts are presented for high resolution refractive index sensing from $n_{\mathrm{H}}=1$ to 1.67 as the nano-holes were readily wetted and filled with various solvents and oils through an intact fibre cladding.

\footnotetext{
${ }^{1}$ Department of Electrical and Computer Engineering, University of Toronto, 10 King's College Rd., Toronto, ON M5S 3G4, Canada. ${ }^{2}$ Department of Physics, Payame Noor University (PNU), P.O. Box: 19395-4697 Tehran, Iran. ${ }^{3}$ These authors contributed equally: Keivan Mahmoud Aghdami, Abdullah Rahnama.

凶email: k_aghdami@pnu.ac.ir; abdullah.rahnama@mail.utoronto.ca
} 
T he compact and flexible format of optical fibre serves broadly as a high-capacity conduit in today's information highway. These advantages further facilitate optical sensing of the local environment that can reach over short to long distances and probe into challenging environments ${ }^{1,2}$. By the nature of strong optical confinement, standard fibres require micro- or nano-engineering of devices directly inside of the core waveguide that is frequently applied by means of chemical etching ${ }^{3}$, mechanical polishing ${ }^{4}$, thermal tapering ${ }^{5,6}$, laser modification ${ }^{7,8}$, or ion milling 9 . Such structures redirect the waveguiding light to probe outside of the fibre cladding. However, optical probing is significantly more responsive when the external environment can be brought close to or directly into the fibre core as provided by photonic bandgap hollow-core fibre. The open structure enables refractive index (RI) sensing of gases ${ }^{10}$ and liquids ${ }^{11,12}$ but without the advantages for remote, localised or distributed sensing that is otherwise possible when micro-devices such as open cavities, micro-optical resonators and interferometers ${ }^{13}$ have been embedded inside of a solid core fibre. Such micro-devices enable localised points of optical sensing at the core waveguide by basic means of absorption, fluorescence, scattering (Rayleigh, Mie, and Brillouin), diffraction, and interferometry ${ }^{14-18}$. These internal elements improve optical sensitivity that enables broadly based lab-in-fibre applications in biology ${ }^{19}$ or healthcare ${ }^{20}$ such as label-free detection of cancer biomarkers ${ }^{21}$.

Fibre Bragg gratings (FBG) are one favoured device in localised optical fibre sensing owing to sharp and environmentally responsive resonances ${ }^{3}$. The FBG further benefits from the ease of external laser fabrication through the cladding core ${ }^{22}$. However, the FBG typically probes the local environment through the cladding, without benefiting from the open photonic bandgap structure such as available in silicon photonic or another planar optical circuit technologies ${ }^{17,23-25}$. An open periodic structure with high RI contrast offers strong photonic bandgap responses, which can be spectrally and spatially narrowed by optical defects to provide high-Q micro-resonators for highly localised sensing. Such micro-resonators enable optical trapping ${ }^{26}$, label-free sensing ${ }^{27}$ or single nano-particle detection ${ }^{28}$ on a chip, but have been limited in fibre by a solid core FBG buried deeply in the cladding. Various means of chemical, laser, and mechanical machining or thinning of the cladding have facilitated evanescent sensing at the surface of the $\mathrm{FBG}^{14,29-31}$. An open-structured photonic crystal has otherwise been challenging to fabricate transversely into the fibre core. Ion milling or laser machining at the core of a thinned fibre has provided FBG sensors with nanostructured surface relief ${ }^{32,33}$, blind holes $^{34}$, and through holes $^{35,36}$. However, the cladding processing renders such fibres mechanically fragile and lacking robustness for practical application. Yang et al. $^{37}$ accessed the core waveguide through a narrow cladding channel to open an array of micro-holes by chemical etching of self-focused laser tracks. Third-order Bragg stopbands provided a microfluidic RI-sensing response, but were restricted to the low sensitivity of $5 \mathrm{~nm} / \mathrm{RIU}$ and narrow RI window from $1.32-1.41$ owing to a large hole diameter $(1.35 \mu \mathrm{m})$ exceeding the optical wavelength.

Femtosecond lasers provide an alternative direction for opening high-aspect-ratio holes in transparent materials by a singlepulse nano-explosion from a filament-shaped beam focus. Hole diameters of $\sim 200 \mathrm{~nm}$ diameter have been demonstrated in glasses by Kerr lensing, and axicon optics ${ }^{38-43}$. Our group has further harnessed surface aberration of glass plates to form similarly long filament tracks in bulk glass ${ }^{44}$. This approach was extended into optical fibre by using RI-matching fluid to eliminate astigmatism of the cylindrical fibre shape and generate filament arrays having first-order Bragg stopbands ${ }^{45-47}$.
In this work, the laser interaction was further scaled up to drive a controlled filament explosion in the fibre cross-section, resulting in densely packed arrays of uniform nanoholes ${ }^{48}$. The isolated, blind or through holes were patterned with controllable positioning to selectively pierce the core and/or cladding and enabled strong photonic stopbands to be formed directly in the core waveguide with minimal processing of the surrounding cladding. FBGs with strong resonances and sharply resolved $\pi$-shifts are presented that provided high capillarity for wetting with various solvents and oils, demonstrating high-resolution RI sensing from $n_{\mathrm{H}}=1-1.67$ through an intact fibre cladding.

\section{Results}

Nanohole FBG-modelling stopbands. A schematic representation of the nanohole array formation by laser filament nanoexplosion is depicted in Fig. 1. The approach meets four key challenges for nano-structuring of strong photonic stopbands in optical fibre. First, the beam delivery avoids astigmatic aberration by the cylindrical cladding shape (Fig. 1), resulting in the formation of nanoholes partially to fully through the fibre crosssection, without thinning or inducing deleterious damage in the fibre cladding structure. Second, the nanohole processing facilitates nanohole assembly on small periodicity at optical wavelength scale, without inducing melting or significant heat-affected zone. The resulting 0.46 contrast in RI provides strong photonic bandgap responses without high optical scattering loss. Third, the laser direct writing affords flexible and rapid patterning, to tune the spectral response and provide micro-cavity like responses such as $\pi$-shifted FBGs. Chemical etching facilitates photonic bandgap engineering by tuning the nanohole diameter from $200 \mathrm{~nm}$ to $700 \mathrm{~nm}$. And fourth, the nanoholes draw significant capillary force to enable wetting by a wide range of solvent types fully through the $125 \mu \mathrm{m}$ diameter of the fibre (Supplementary Movie 1,2). The nanohole array thus defines an open and flexible FBG-sensing structure that can be fabricated rapidly in a singlestep procedure, and provide robust mechanical integrity together with strong optofluidic responses due to the subwavelength hole diameter.

The anticipated optofluidic responses of the nanohole FBG structure (Fig. 1) are significantly stronger and more sensitive than with the traditional FBG grating as demonstrated by the simulations in Fig. 2. The second-order grating responses were modelled in standard telecommunication fibre (SMF-28) having $300 \mathrm{~nm}$ diameter holes with $1072 \mathrm{~nm}$ periodicity and a relatively short length of $643 \mu \mathrm{m}$. The potential for precise RI sensing is

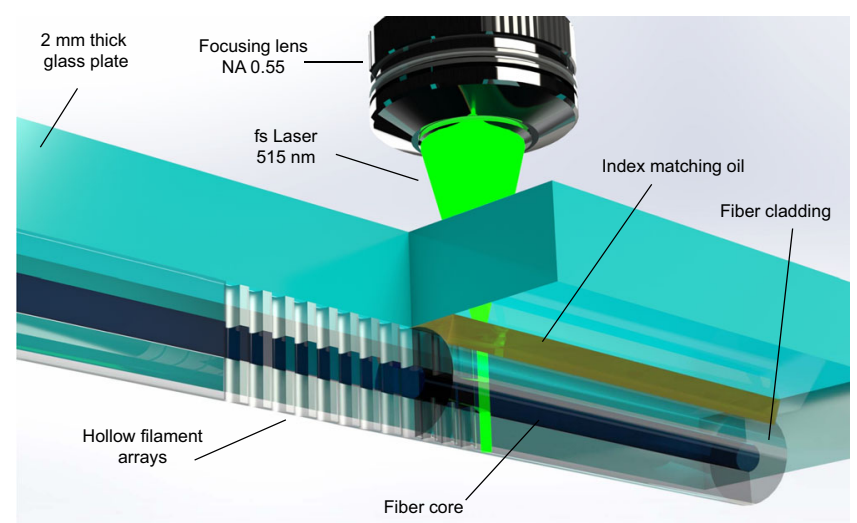

Fig. 1 Femtosecond laser filament processing in optical fibre. Schematical arrangement of optical aberration plate and optical fibre used for generating femtosecond laser filaments, and opening high-aspect ratio nanoholes through the fibre cladding and core waveguide. 

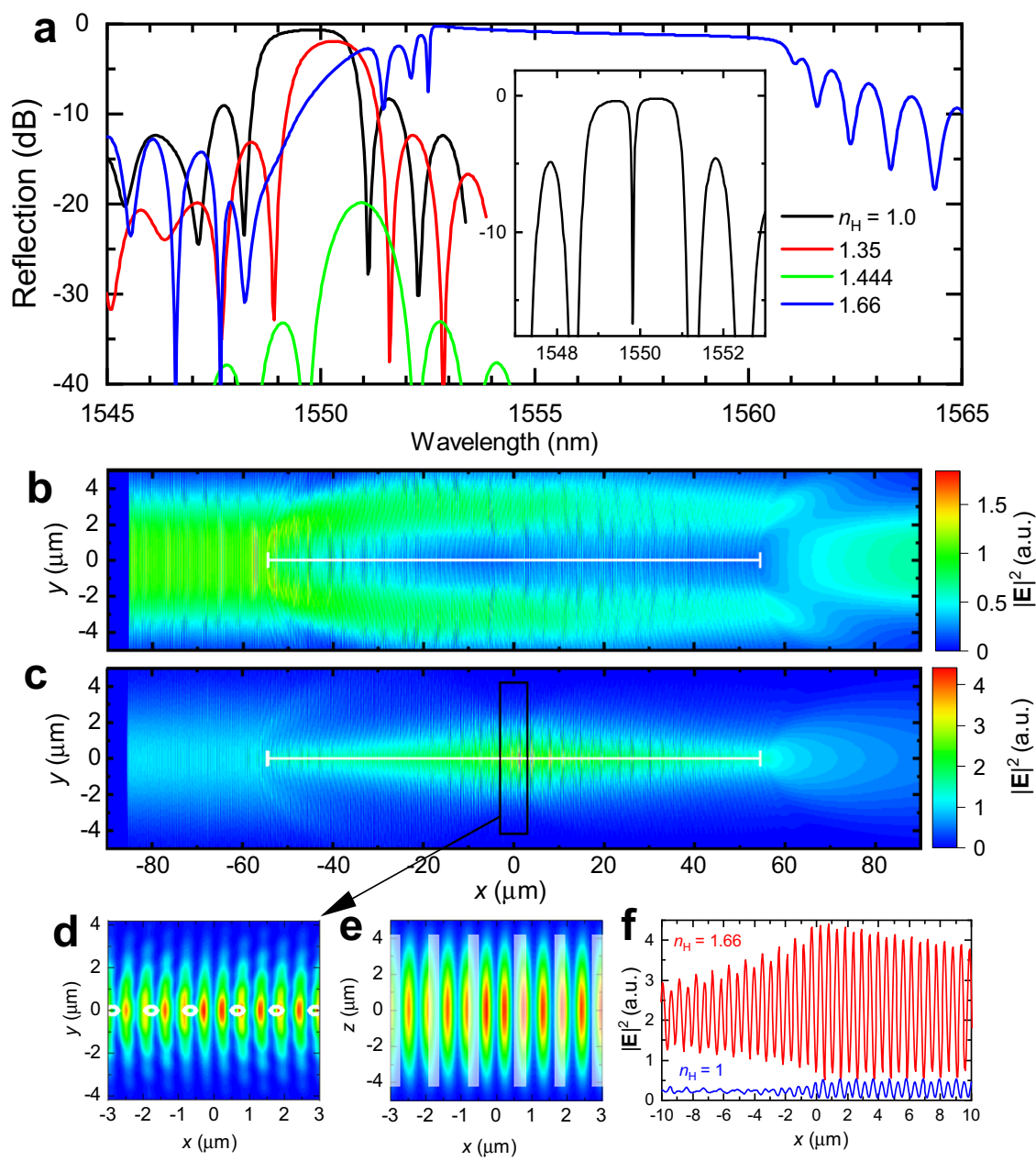

Fig. 2 Simulated spectra and light-field distribution inside of the nanohole arrays. Simulated reflection spectra (a) for a second-order FBG of uniformly pitched nanoholes $(\Lambda=1.072 \mu \mathrm{m})$ having $300 \mathrm{~nm}$ diameter, $0.643 \mathrm{~mm}$ array length ( 600 holes) and high RI contrast $(\Delta n=-0.45$ to +0.21$)$ when filled with air, solvent or oil. A sharply resolved transmission band (30 pm FWHM) has opened (inset spectrum, $n_{H}=1$ ) from a $\pi$-shifted FBG of 1200 holes. The simulated intensity distribution transverse to nanoholes (xy plane) for low $\left(n_{H}=1.0\right)(\mathbf{b})$ and high $\left(n_{H}=1.66\right)(\mathbf{c})$ RI cases of a $\pi$-shifted FBG (100 holes, $x=-53.6$ to $+53.6 \mu \mathrm{m})$. Magnified views for the high RI case reveal intensity concentration in the filament plane $(\mathbf{e})$ and around the $\pi$-defect $(\mathbf{d} ; x=0)$. White circles and bars outline the hole positions. Axial intensity oscillation ( $x$ axis, $y=z=0$ ) comparing (f) high and low RI cases.

noted by the strong and rapidly shifting stopbands (Fig. 2a) when nanoholes are filled with air and solvents having different RI values spanning from $n_{\mathrm{H}}=1-1.66$ (see also Supplementary Fig. 1). The subwavelength hole diameter provides increasingly stronger (i.e., $>-2 \mathrm{~dB}$ for air) and broader resonances (up to $7 \mathrm{~nm}$ bandwidth), rising up for both increasing or decreasing RI from the matching condition at $n_{\mathrm{H}} \cong n_{\text {eff }}=1.450$ (Fig. 2a). With variable hole diameter, the EME modelling indicated a high RI sensitivity response of up to $600 \mathrm{~nm} / \mathrm{RIU}$ will be available at high RI (i.e., $n_{\mathrm{H}}=1.66$ ) and a large hole diameter of $700 \mathrm{~nm}$.

When imposing a $\pi$-shifted defect into the nanohole array, the simulated stopband (Fig. 2a, inset spectrum) opens a sharp and narrow transmission resonance $(\Delta \lambda=30 \mathrm{pm}, 3 \mathrm{~dB}$ bandwidth) inside of the air-filled stopband, representing a moderately strong resonator quality factor $\left(Q \sim 10^{4}\right)$. The strong influence of the $\pi$ defect and nanohole array on the guided light-field distribution is demonstrated by the high repulsion (Fig. 2b) or attraction (Fig. 2c) of the mode within a narrow $(\sim 2.5 \mu \mathrm{m})$ zone following along nanoholes filled with air $\left(n_{\mathrm{H}}=1\right)$ or oil $\left(n_{\mathrm{H}}=1.66\right)$, respectively. For the higher RI case $\left(n_{\mathrm{H}}=1.66\right)$, magnified views of the intensity profiles near the $\pi$-defect $(x=0)$ reveal an asymmetric narrowing from the fundamental mode field from $10.4 \pm 0.5 \mu \mathrm{m}$ diameter to $5 \mu \mathrm{m}$ (Fig. $2 \mathrm{~d}$ ) and $8 \mu \mathrm{m}$ (Fig. 2e) for respective perpendicular and parallel directions with respect to the hole axis. The beginning of a micro-cavity response is noted along the fibre centre axis (Fig. $2 \mathrm{f}$ ) by the light intensity drawing into a short $66 \mu \mathrm{m}$ zone (full $3 \mathrm{~dB}$ ) as presented for the air $\left(n_{\mathrm{H}}=1\right)$ and oil $\left(n_{\mathrm{H}}=1.66\right)$ cases (Fig. $\left.2 \mathrm{f}\right)$.

Nanohole array FBG-morphology. Long and uniform laser filament shapes (Fig. 1) of up to $125 \mu \mathrm{m}$ length were provided by surface aberration from glass plates of up to $3 \mathrm{~mm}$ thickness. In this arrangement, high laser pulse energies of $7 \mu \mathrm{J}$ drove a uniform nano-filament explosion without distortion from Kerr lens focusing or plasma defocusing effects. The nonlinear optical benefits of narrowing modification dimensions below diffractionlimited sizes were thus retained. Isolated, blind, and through holes could be tailored by the laser and focussing controls to any position in the fibre cross-section. An example of an FBG with nanohole arrays reaching fully through the cladding and core is presented in Fig. 3. A schematic of the FBG (centre image) represents the dense packing of the nanoholes on $1.072 \mu \mathrm{m}$ periodicity, targeting a high-aspect ratio of processing control reaching through the $125 \mathrm{~nm}$ diameter fibre cladding. The evidence for this high-aspect-ratio hole geometry is provided in the 


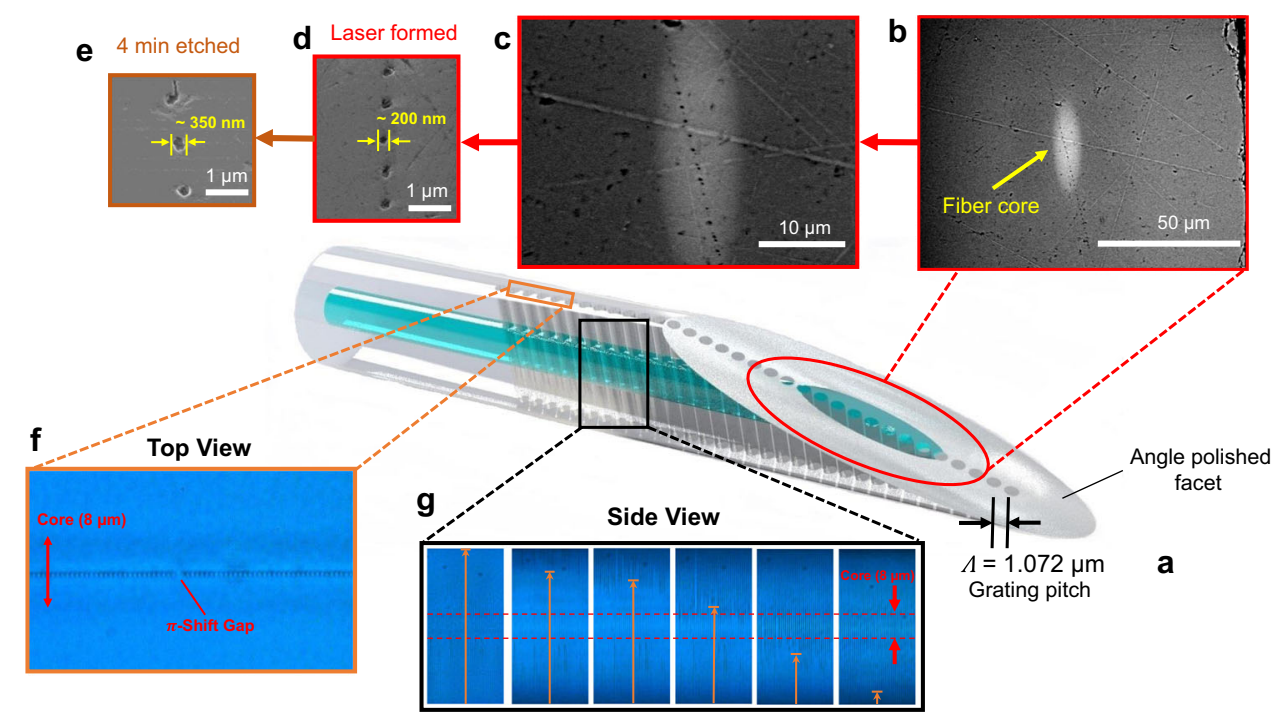

Fig. 3 Morphological characterisation of the nanoholes with SEM and optical microscopy images. Schematical view of a nanohole embedded optical fibre with angled facet (a) and supporting microscopic imaging ( $\mathbf{b}-\mathbf{g})$. Optical microscopy of the nanohole array showing the central alignment of the array to the core in the end view (f) and a time sequence (left to right) of evaporating isopropanol with menisci level (yellow arrow) moving downward in side views of the nanoholes $(\mathbf{g})$. Under increasing magnification, SEM images of the angled fibre facet (a-d) unveil open nanoholes having formed continuously and uniformly ( $200 \mathrm{~nm}$ diameter) through the core (white oval zone) and cladding without a breakthrough on the tight $1.072 \mu \mathrm{m}$ periodic spacing. SEM (e) shows the hole diameter expanded to $350 \mathrm{~nm}$ after 4 min of chemical etching (5\% HF).

time sequence of microscope images showing the evaporation of isopropanol from fully wetted (i.e., air-filled) nanoholes, recorded over $\sim 10$ s (see also Supplementary Movie 1,2). A wide range of solvent types was found to readily wet and fill the nanoholes of such laser structured fibre.

The provision of RI-matching oil around the fibre cladding (Fig. 1) did not inhibit near-neighbour filament nor nanohole formation. Lateral damage zones were insignificant both at surfaces and the internal volume such that isolated holes could be formed, pulse by pulse, in arrays having periods as small as $1.072 \mu \mathrm{m}$ (Fig. 3f, g). The point-by-point writing permitted the insertion of optical defects such as the $\pi$-shift identified in the optical microscope image (Fig. 3f). The end-view of the nanochannel array, formed on $1.072 \mu \mathrm{m}$ periodicity confirmed a submicron lateral resolution of hole positions inside the core waveguide, and extended well beyond the beam depth of focus $(1.4 \mu \mathrm{m})$ to reach through the fibre cladding cross-section (Fig. 3g).

The cross-sectional morphology of the nanohole structures was further examined by scanning electron microscope (SEM) imaging. A section of nanohole FBG was cleaved and polished at an oblique angle $\left(\sim 30^{\circ}\right)$, as shown schematically in Fig. $3 \mathrm{a}$. Electron microscopy of the oblique fibre cross-section identifies an array of nanoholes having formed through the core and cladding (Fig. 3b). With an increasing magnification of the core section, the sequence of SEM images (Fig. 3b-e) confirm the formation of isolated nanoholes having a relatively uniform cylindrical shape, and positioned on $\Lambda=1.072 \mu \mathrm{m}$ period with an estimated hole diameter of $\sim 200 \mathrm{~nm}$. A slightly irregular hole perimeter is noted with an internal surface roughness of $\sim 50 \mathrm{~nm}$, which is $\sim 30 \times$ smaller than the optical probe wavelength. Hence, the holes can form fully isolated in tightly packed arrays having nearly uniform cross-section through the core (Fig. 3) and a majority of the cladding. The nanohole arrays are only noted to break into each at the cladding surface over a depth of $\sim 15 \mu \mathrm{m}$. Hence, nanoholes with a high-aspect ratio $>500 \times$ have been demonstrated in the fibre. The present results have not unveiled the high-stress zones expected to be forming around the nanoholes as seen previously ${ }^{42,49}$ in bulk glass when formed with pulses of longer duration (1 ps). Such densified zones may form a barrier to micro-crack formation to toughen the glass in the same way chemical treatment stressing works in display glass. A tensile proofing test of mechanically stripped fibre with and without the nanohole array was completed ${ }^{50,51}$, providing the cumulative failure probability for fracture as shown in Supplementary Fig. 2. As expected, there was only a modest decrease in the strength of the nano-structured fibre, falling to $\sim 30 \%$ of the level for mechanically stripped fibre.

Emersion of the FGBs in diluted hydrofluoric (HF) acid (5\%) provided a second processing step to reproducibly increase the hole diameter, for example, to $\sim 350 \mathrm{~nm}$ diameter after $4 \mathrm{~min}$ (Fig. 3e). Such diameter tuning opens a means for tuning the photonic stopband response, as well as increasing the FBG sensitivity to RI changes.

Photonic stopband response. The validation of strong and responsive photonic stopbands is presented in the reflection spectra of Fig. 4a for nanohole arrays filled with air and a range of solvents (Table 1). Under high RI contrast with air $\left(n_{\mathrm{H}}=1.0\right)$ or Oil10 $\left(n_{\mathrm{H}}=1.66\right)$, the nanohole array provided broad $(\sim 2 \mathrm{~nm})$ and strong $(\sim 3 \mathrm{~dB})$ Bragg resonances with only 600 holes (i.e., $643 \mu \mathrm{m}$ length). The evolution of the spectral profile, reflection peak and linewidth with the increasing grating length for the case of air-filled holes (Supplementary Fig. 3) followed the growth trends of traditional FBGs, expect to develop much more rapidly owing to a $\sim 100$-fold higher RI contrast $(\Delta n=0.45)$ over traditional FBGs $\left(\Delta n \sim 10^{-3}\right)^{2}$. Although the $200 \mathrm{~nm}$ hole diameter encompassed only a $\sim 2 \%$ overlap with the modal field (MFD $\cong$ $10.4 \mu \mathrm{m})$, the gratings provided a strong effective coupling strength of up to $\kappa_{\mathrm{AC}}=9.17 \mathrm{~cm}^{-1}$. The strong responses are enabled by the strong field repulsion or attraction effects of the guided light into or out of the planar grating zone (Fig. $2 \mathrm{~b}$ versus Fig. 2c) for the cases of low and high RI, respectively. As a result, the Bragg resonance had shifted definitively from $1549.93 \mathrm{~nm}$ in the air $\left(n_{\mathrm{H}}=1.0\right)$ to $1551.76 \mathrm{~nm}$ in Oillo $\left(n_{\mathrm{H}}=1.661\right)$, providing an average RI sensitivity of $2.75 \mathrm{~nm} / \mathrm{RIU}$ across a wide RI-sensing range. The progression of strong-to-weak stopbands, from 


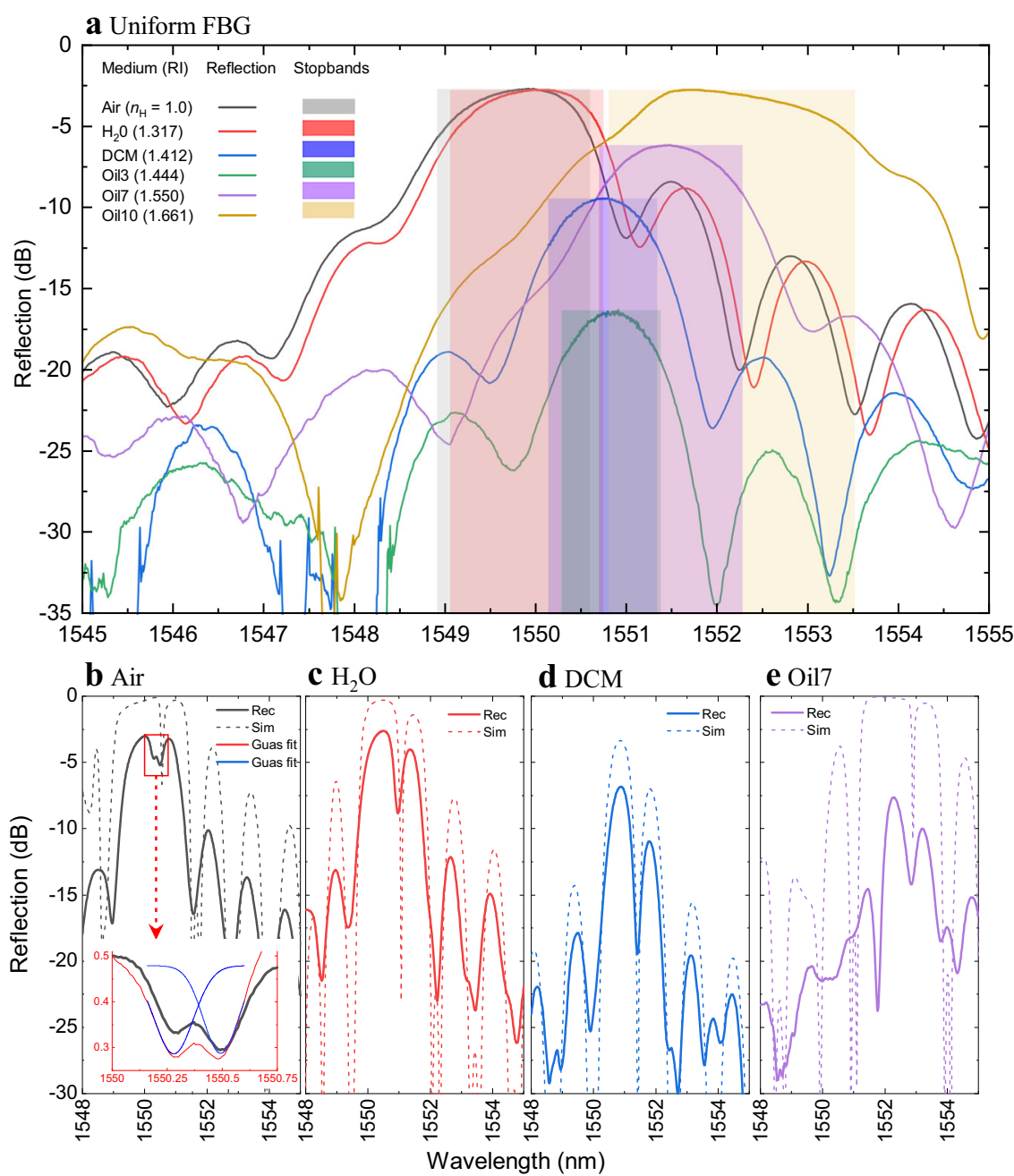

Fig. 4 Photonic stopband response of the FBGs filled with different materials. Reflection spectra (a) recorded from a uniform FBG of 600 nanoholes $\left(E_{\text {pulse }}=4.5 \mu \mathrm{J}, \Lambda=1072 \mathrm{~nm}, 2 \mathrm{~mm}\right.$ plate) forming strong ( $\left.3 \mathrm{~dB}\right)$ and wide $(\sim 2 \mathrm{~nm})$ stopbands when filled with low $\left(n_{H}=1\right)$ or high $\left(n_{H}=1.661\right)$ refractive index materials. The coloured bands mark the $3 \mathrm{~dB}$ bandwidth. Refractive index matching oil $\left(n_{H}=1.448\right)$ dramatically weakens the stopband. Reflection spectra (solid lines) of unpolarized light (b-e) were recorded from a $\pi$-shifted FBG of 1200 nanoholes for four different examples of RI values ( $n_{H}$ by colour from legend $\mathbf{a}$ ) and comparison with simulated spectra for $P$-polarised light (dashed lines). Laser-induced birefringence of $\delta \lambda=210 \mathrm{pm}$ is resolved for the air-filled case ( $\mathbf{b}$ inset). Gaussian line fits of the $\pi$-defects (blue and red lines in $\mathbf{b}$ inset) provide \pm 10 pm spectral precision.

$-2.72 \mathrm{~dB}$ reflection with air to $-17.28 \mathrm{~dB}$ with Oil5 $\left(n_{\mathrm{H}}=1.448\right)$, marks the matching condition on RI, beyond which higher RI (i.e., Oil10, $n_{\mathrm{H}}=1.661$, Fig. 4a) regenerated strong stopbands $(-2.76 \mathrm{~dB})$.

Although strong in reflection, the breadth of the stopbands under high RI contrast is limiting in specifying the precise centre Bragg wavelength. A more rewarding direction was obtained by implanting a $\pi$-defect into a Bragg grating array of 1200 nanoholes, opening well-resolved passbands (Fig. 4b-e, Supplementary Fig. 7). In the progression for nanoholes filled with air, water, dichloromethane and Oil7 $\left(n_{\mathrm{H}}=1.0,1.316,1.412\right.$, and 1.550 in Fig. $4 \mathrm{~b}-\mathrm{e}$, respectively), the $\pi$-defect provided the highest contrast $(>10 \mathrm{~dB})$ and sharpest resolution $\left(\delta \lambda_{\mathrm{B}}=150 \mathrm{pm}\right)$ passbandwidth $(3 \mathrm{~dB})$ when tuned nearer to the refracting index matching condition, $n_{\mathrm{H}} \cong n_{\mathrm{G}}$ (Fig. $4 \mathrm{~d}$ and see also Supplementary Fig. 7a). For air-filled nanoholes, the resonance is separated into two $\sim 3 \mathrm{~dB}$ peaks $\left(\delta \lambda_{\mathrm{B}}=210 \mathrm{pm}\right.$, Fig. $\left.4 \mathrm{~b}\right)$, unveiling a waveguide birefringence of $\Delta n_{\mathrm{H}}=0.07$. Otherwise, the birefringence was unresolved and served only to broaden the passband $\left(\delta \lambda_{\mathrm{B}}=150-400 \mathrm{pm}\right)$ with increasing $\mathrm{RI}$ contrast examined in the range $1.31<n_{\mathrm{H}}<1.67$ (Supplementary Fig. 8a). The point-bypoint writing of such high-aspect ratio nanoholes with $<100 \mathrm{~nm}$ positional precision thus facilitated photonic band shaping with sharp spectral features tuned to $\sim 100$ pm resolution.

EME modelling, (Fig. 4b-e, dashed line spectra) provided a good representation of the $\pi$-shifted grating reflection (Fig. $4 \mathrm{~b}-\mathrm{e}$, solid line spectra) with the hole diameter optimised to $\sim 200 \pm 10 \mathrm{~nm}$ for the 1200 nanohole array design $(\Lambda=1.072 \mu \mathrm{m})$. The simulated spectra have reproduced the broad stopband and sideband features as well as the narrow $\pi$ defect response and accurately tracked the wavelength shifts for the full range of RI changes from $n_{\mathrm{H}}=1.0$ (Fig. $4 \mathrm{~b}$ ) to 1.550 (Fig. 4e). However, the grating reflection fell short of the simulated peak values by $3 \mathrm{~dB}$ to $7 \mathrm{~dB}$ over the respective air $\left(n_{\mathrm{H}}=1.0\right)$ to Oil7 $\left(n_{\mathrm{H}}=1.550\right)$ cases, pointing to unaccounted losses from Rayleigh scattering on nanohole surface roughness $(\sim 50 \mathrm{~nm}$, Fig. 3), variances of up to $\pm 50 \mathrm{~nm}$ on the nanohole positioning, and first-order Bragg radiation.

To improve on the resolution for RI sensing, Gaussian-shaped functions were found to best match the $\pi$-defect transmission dip, for example, as shown in the case of the air-filled holes (Fig. $4 \mathrm{~b}$ inset). The spectral matching enabled centre resonant wavelengths to be specified with a precision of $\pm 10 \mathrm{pm}$. Otherwise, the Gaussian representations furnished linewidths varying from 180 


\begin{tabular}{|lll|}
\hline \multicolumn{2}{l}{$\begin{array}{l}\text { Table } \mathbf{1} \text { Refractive index (RI) of liquids applied inside of } \\
\text { nanohole FBGs. }\end{array}$} & \\
Medium & Abbreviation & RI at $\boldsymbol{\lambda}=\mathbf{1 5 5 0} \mathbf{~ n m}^{\mathbf{5 5}}$ \\
\hline Air & Air & 1.002 \\
Water & $\mathrm{H}_{2} \mathrm{O}$ & 1.3164 \\
Methanol & MeOH & 1.3174 \\
Acetone & Ace & 1.3483 \\
Isopropanol & IPA & 1.3737 \\
Dichloromethane & DCM & 1.4124 \\
Cargile AA-1.450 & Oil1 & 1.440 \\
Cargile AA-1.452 & Oil2 & 1.442 \\
Cargile AA-1.454 & Oil3 & 1.444 \\
Cargile AA-1.456 & Oil4 & 1.446 \\
Cargile AA-1.458 & Oil5 & 1.448 \\
Cargile A-1.540 & Oil6 & 1.522 \\
Cargile A-1.572 & Oil7 & 1.550 \\
Cargile A-1.586 & Oil8 & 1.562 \\
Cargile A-1.630 & Oil9 & 1.600 \\
Cargile B-1.700 & Oil10 & 1.661 \\
Cargile M-1.705 & Oil11 & 1.670 \\
\hline
\end{tabular}

to $360 \mathrm{pm}$ (FWHM) for the unresolved birefringence cases (Fig. 4c-e; see also Supplementary Fig. 7).

Photonic bandgap engineering - etching. The as-formed nanoholes provided added utility in guiding chemical etchant to tune the hole diameters and further engineer the stopband response. SEM revealed a near-linear response of increasing hole diameter with etching time, beginning from the $\sim 200 \mathrm{~nm}$ diameter for the laser-formed nanohole and expanding to $350 \mathrm{~nm}$ for the case of 4 min etching in 5\% HF acid (Fig. $3 \mathrm{e}$ ). In real-time monitoring of FBG reflection spectra (1200 holes), the stopbands in both uniform and $\pi$-shifted gratings (Supplementary Fig. 4a) shifted monotonically to a shorter wavelength over a 7 min etching time. The blue spectral shifting arises from a drop in effective RI as the lower RI of etchant $\left(n_{\mathrm{H}} \cong n_{\mathrm{H} 2 \mathrm{O}} \cong 1.3164\right)$ displaces the higher RI glass $\left(n_{\mathrm{G}}=1.45\right)$. Increasing hole diameter is noted to weaken and narrow the stopbands (Supplementary Fig. $4 \mathrm{~b}$ ) from -3.7 to $-29 \mathrm{~dB}$ in strength and $1.5-0.97 \mathrm{~nm}$ in bandwidth, over the 7 min etching time. However, the $\pi$-shifted passband (not plotted) retained a full $9 \mathrm{~dB}$ contrast inside of the FBG stopband, even as the stopband weakened by $>20 \mathrm{~dB}$ over the full 7-min etching time (Supplementary Fig. 4b). The preservation of sharply resolved $\pi$-defect resonances attests to a highly ordered and unbroken patterning of the high-aspect ratio nanoholes, that does not breakthrough on a tight packing density $(1.072 \mu \mathrm{m}$ period) even as diameters were opened up over the 7 min etching time.

Nanohole FBGs with uniform gratings were prepared with different etching times $(0,2,4$, and $6 \mathrm{~min})$ to provide a widely varying FBG response (Supplementary Fig. 5) when filled with solvents or oils spanning a large range of RI values (Table 1). The nanohole arrays as formed by the laser $(0 \mathrm{~min})$ typically offered the strongest stopbands (Fig. 5a). The progression to weaker stopbands (Fig. $5 \mathrm{a}-\mathrm{c}$ ) varied from strongly to weakly for solvents having the lowest (Fig. $5 \mathrm{a}, n_{\mathrm{H}}=1$ ), matched (Fig. $5 \mathrm{~b}, n_{\mathrm{H}}=1.448$ ) and highest (Fig. $5 c, n_{\mathrm{H}}=1.6$ ) values of RI. This progression is noted in the plot of peak Bragg reflectance (Fig. 5d) over the full range of tested RI values (Table 1). The fall-off was most pronounced for air $\left(n_{\mathrm{H}}=1\right)$, decreasing by $>25 \mathrm{~dB}$ over the $6 \mathrm{~min}$ etching time. The fall-off was delayed in the case of higher RI oils with RI values of $n_{\mathrm{H}}=1.522-1.66$, where reflectivity first increased to a maximum of $\sim 5 \mathrm{~dB}$ for $2-4$ min etching time. In all cases, the stopbands were weakest for the $6 \mathrm{~min}$ etching time. The bandwidth $(3 \mathrm{~dB})$ of the Bragg stopbands was also strongly influenced by the chemical etching time, either narrowing by $\sim 50 \%$ or broadening by more than threefold according to the negative $\left(n_{\mathrm{H}}<1.45\right)$ or positive $\left(n_{\mathrm{H}}>1.45\right)$ contrast of RI (Fig. 5e).

The alignment of the EME-modelled spectra (Supplementary Fig. 1) with the observed reflection spectra (Fig. 4, Supplementary Fig. 5) provided a precise, semi-empirical estimate of the nanohole diameter, for example, yielding $500 \pm 20 \mathrm{~nm}$ diameter for 4 min etching time (Supplementary Fig. 6). In this way, hole diameters of $220,300,500$, and $700 \mathrm{~nm}$ were assigned with variances of $\sim \pm 10 \mathrm{~nm}$ to FBGs opened with $0,2,4$, and $6 \mathrm{~min}$ etching time, respectively. The spectral corroboration identifies a peak value of FBG reflectance (Supplementary Fig. 4, Fig. 5d) arising on the first quarter wavelength resonance of the nanohole diameter (i.e., $\lambda / 4 n_{\mathrm{H}}$ ), for example, encompassing hole diameters of 220-300 nm for solvents varying from $n_{\mathrm{H}}=1$ for air to $n_{\mathrm{H}}=1.41$ for Dichloromethane. The steep fall-off of reflection thus aligns with an anti-resonance on doubling of the hole diameter to $\lambda / 2 n_{\mathrm{H}}$, corresponding to diameters of $500-700 \mathrm{~nm}$ for air to high index oil $\left(n_{\mathrm{H}}=1\right.$ to 1.60$)$. Hence, the diameter of nanoholes transitions from a first-order resonance for the strongest stop reflection band (0-2 min etching) to the first anti-resonance $(6 \mathrm{~min})$ over the ranges of laser formation and chemical etching tested here.

An increasing hole diameter further provided stronger spectral shifts of the stopbands (Supplementary Fig. 5, Fig. 5a-c), moving in reversed directions as expected depending on the positive $\left(n_{\mathrm{H}}>n_{\mathrm{G}}\right)$ or negative $\left(n_{\mathrm{H}}<n_{\mathrm{G}}\right)$ contrast of solvent RI with respect to the glass index. The largest hole diameter $(\sim 700 \mathrm{~nm}$ for $6 \mathrm{~min}$ ) and highest RI oils offered the highest wavelength sensitivity, with the Bragg centre wavelength shifting by up to $+40 \mathrm{~nm}$ for $n_{\mathrm{H}}=1.60$ solvent over the 0 to $6 \mathrm{~min}$ etching time. In contrast, a smaller and negative wavelength shift of $-2.2 \mathrm{~nm}$ was noted (Fig. 5a) for the case of the air-filled holes over the same 6 min etching time. Under index matching (Fig. $5 \mathrm{~b}, n_{\mathrm{H}}=1.45$ ), the Bragg resonance did not shift.

High-resolution RI sensing. In order to provide the highest resolution RIU sensing, $\pi$-shifted FBGs of 1200 hole arrays were modified with similar etching times (0, 2, 4, and $6 \mathrm{~min})$. EME modelling offered close matching of the spectra strong bands, side lobes, and $\pi$-shift passbands (Fig. $4 \mathrm{~b}-\mathrm{e}$ ), yielding similar values of effective hole diameters, corresponding to $220,300,500$, and $700 \mathrm{~nm}$ for the respective $0,2,4$, and 6 min etching times. The $\pi$ defect passbands (Supplementary Fig. 7) were spectrally fitted to Gaussian line shapes, varying from 200 to $400 \mathrm{~nm}$ linewidth (Supplementary Fig. 8). A narrow birefringent splitting $(\sim 200 \mathrm{pm})$ of the $\pi$-defect was occasionally resolved in the spectra (Supplementary Fig. 8) in cases with the strongest stopbands and largest contrast in the RI.

With spectral line fitting (Supplementary Fig. 7) of the $\pi$ defects, centre Bragg wavelengths could be determined to $\pm 10 \mathrm{pm}$ precision, enabling RI sensing to a high-resolution of $10^{-5}$ RIU. When plotted against the RI (Fig. $5 \mathrm{f}$, solid fonts), the $\pi$-resonance shifts demonstrated an impressive RI response of FBG stopbands, shown globally over an extraordinary range of RI values $\left(n_{\mathrm{H}}=1-1.66\right)$ and nanohole diameters $(220-700 \mathrm{~nm})$. The EME modelling (Fig. 5f, dashed line) followed each data set to $\pm 200 \mathrm{pm}$ (rms) spectral precision, relying only on one value of optimised hole diameter across the full RI testing range. Discrepancies in the air $( \pm 500 \mathrm{pm})$ and between methanol and water may arise from surface tensions effects that require further study. Sharply forming $\pi$-defect resonances were identified in all cases except the two highest indexes $\left(n_{\mathrm{H}}=1.661\right.$ and 1.670) and large diameter $(6 \mathrm{~min})$ condition (open triangle Fig. 5f), where stopbands became overly broad and mixed with sidebands (see Supplementary Fig. 5d). 

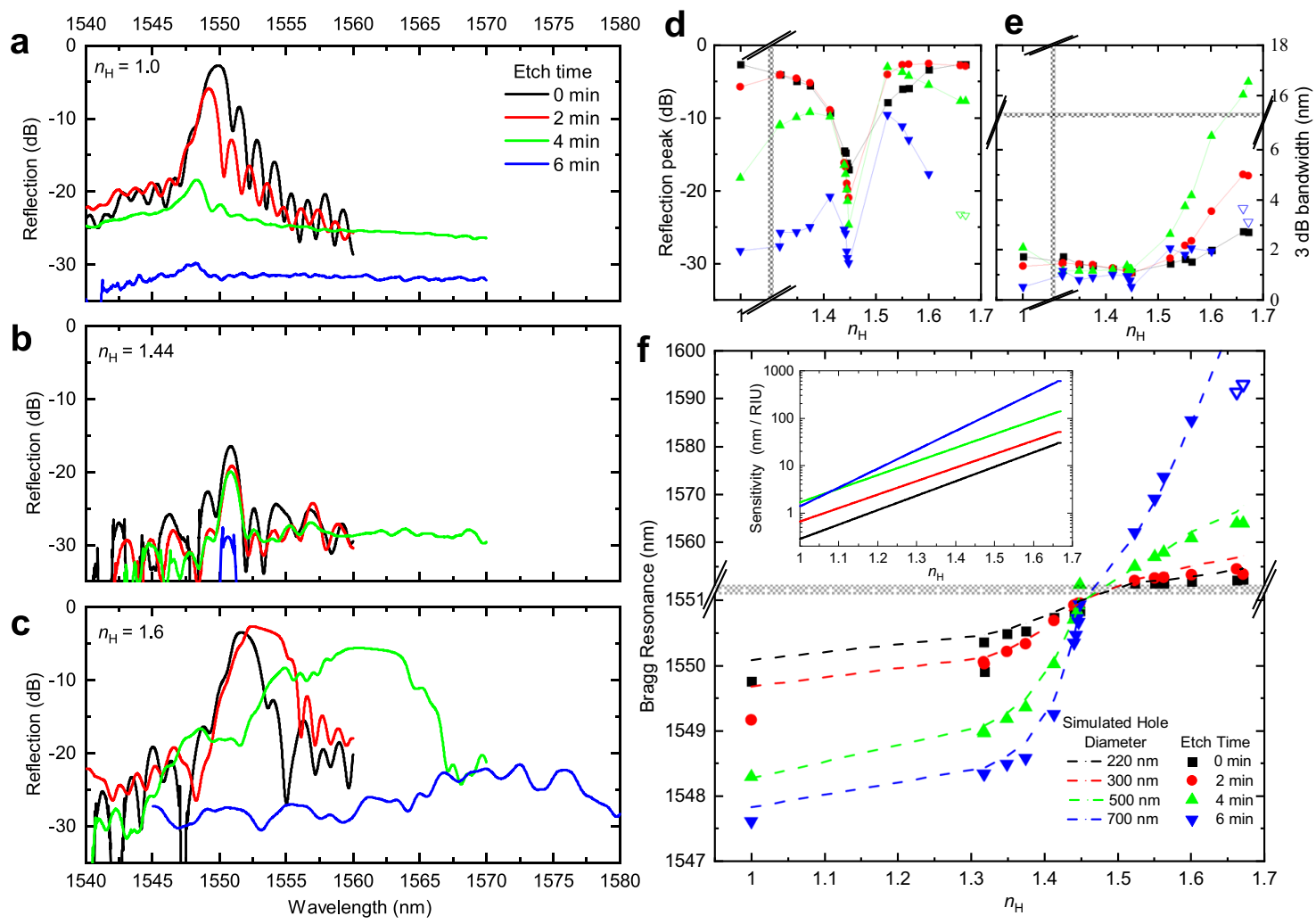

Fig. 5 High-resolution RI sensing enabled by FBGs with different hole diameters. Reflection spectra recorded from uniform FBGs $\left(E_{\mathrm{pulse}}=4.5 \mu \mathrm{J}\right.$, $\Lambda=1072 \mathrm{~nm}, 600$ holes $)$ filled with low $\left(\mathbf{a} n_{H}=1\right)$, medium $\left(\mathbf{b} n_{H}=1.44\right)$, and high $\left(\mathbf{c} n_{H}=1.661\right)$ refractive index materials, revealing the influence of increasing nanohole diameter due to varying chemical etching time (0-6 min). Peak reflection strength (d) and 3-dB linewidth (e) of the Bragg resonances plotted as a function of refractive index for different chemical etching times (following legend a). Bragg resonance wavelength (shifts on right axis) plotted versus refractive index $\mathbf{f}$ for different chemical etching times. A global fit of the data by EME simulation is presented (dashed lines) for hole diameters of 220-700 nm (see legend). The slopes of data provide a strongly increasing refractive index sensitivity (inset) for increasing RI and increasing hole diameter (colour-coded lines). Shaded zones mark axis scale changes.

The narrow $\pi$-shifted stopbands provided strong optical responses to sense the local environment, as demonstrated in the wide $1548-1590 \mathrm{~nm}$ shift in Bragg wavelengths (Fig. 5f). The slopes of these responses yielded a widely ranging response in RIU sensitivity (Fig. 5f, inset) that reached as high as $600 \mathrm{~nm} / \mathrm{RIU}$ for the case of highest RI $\left(n_{\mathrm{H}}=1.66\right)$ and largest hole diameter $(700 \mathrm{~nm})$. This RIU sensitivity is comparable to the best FBGbased demonstrations to date (i.e., $945 \mathrm{~nm} / \mathrm{RIU}$ in ref. ${ }^{52}$ ). Moreover, spectral line fitting $( \pm 10 \mathrm{pm})$ offered a high-resolution determination of the RI to $\pm 10^{-4}$ precision.

The chemical etching of the filaments demonstrated an attractive progression of improving RIU sensitivity response (Fig. 5f, inset), but at the cost of forming broader and weakened stopbands as the hole, the diameter was increased from $200 \mathrm{~nm}$ to $700 \mathrm{~nm}$ (i.e., see Fig. 5 and Supplementary Fig. 5). This range of subwavelength hole diameter (i.e., below $\lambda / n=1.072 \mu \mathrm{m}$ ) was beneficial in circumventing optical resonances in the holes that interfere with the photonic stopbands. In this way, strong stopbands enabled a signal to noise ratio (SNR) amenable for optical sensing over the moderately large range of reported RI values, 1-1.67. Scaling to a larger hole diameter would otherwise diminish the SNR and narrow the RI-sensing range, for example, falling sevenfold to the 1.32-1.41 range as reported in ref. ${ }^{37}$ for $1.665 \mu \mathrm{m}$ hole width. The benefit of the sub-micron hole dimensions is thus narrower and stronger stopbands that avoid ambiguity in sensing multiple Bragg resonances in distributed FBG systems. Moreover, the nanohole FBG avoids a low limit cut off in the RI (i.e., $n \sim 1.48$ ) for total internal reflection as in the case of evanescence-based FBG refractometry ${ }^{14,29,30}$.
The nanohole array presents a responsive optical fibre sensor, retaining cladding strength while readily wetting with numerous solvents that can reach into the open photonic structure formed along with the fibre core. The nanoholes are amenable to hosting a wide range of materials, for example, such as nematic liquid crystals (NLC) ${ }^{53}$. The small, $200 \mathrm{~nm}$ diameter capillaries were found to impose a strong axial molecular alignment of NLC, manifesting in strong optical birefringence in the fibre polarisation modes that facilitated an all-fibre dynamically switchable polarisation filter ${ }^{53}$. The facile means of laser writing and patterning of high-aspect-ratio nanoholes introduced here thus opens the realm for creating strong and compact photonic stopband devices directly in traditional optical fibres while facilitating environmental sensing through a thick robust cladding. The line-by-line writing is an intrinsically fast process, being single-step and scalable to sub-second exposure times with only modest repetition rates of $\sim 1 \mathrm{kHz}$ in comparison with current fabrication techniques ${ }^{37,52}$. The fabrication method is extensible to other types of fibres and fibre materials, or to the structuring of two-dimensional arrays of nanoholes in the fibre ${ }^{54}$. Such structuring would permit the engineering of compact 2D photonic bandgap devices directly inside of the fibre that is attractive for tailoring the coupling to cladding modes or in and out of the fibre through the radiation modes 46,54 . In this way, the minimally invasive methods of filament explosion and chemical etching are enabling in the photonic bandgap engineering of traditional optical fibre, promising to transform how fibres shape the flow of light and sense the local environment from applications in biomedical probes through to large area communication networks. 


\section{Methods}

Laser fabrication of hollow-filament arrays. A frequency-doubled Yb-doped fibre laser (Amplitude Systems, Satsuma) provided femtosecond pulses of $515 \mathrm{~nm}$ wavelength, $250 \mathrm{fs}$ pulse duration and $\mathrm{M}^{2}<1.2$ beam quality. The $800 \mathrm{kHz}$ repetition rate was down counted to $1 \mathrm{~Hz}$, to provide up to $8 \mu \mathrm{J}$ pulse energy to the filament-forming exposure arrangement (Fig. 1). The laser beam was expanded $\sim 2$ times (Linos Magnification Beam Expander, 4401-257-000-20) to fill the full aperture of an aspherical focusing lens (New Focus, 5722-A-H) of 0.55 NA. A $0.8 \mu \mathrm{m}$ spot radius $\left(1 / \mathrm{e}^{2}\right.$ intensity) and $1.4 \mu \mathrm{m}$ depth of the focus is expected if focussed directly into silica glass.

In the arrangement of Fig. 1, a long and uniform filament beam shape with a diameter of $<1 \mu \mathrm{m}$ was generated by surface aberration induced by the flat surfaces of fused silica plates (Nikon, NIFS-S (S-grade)) positioned between the lens and optical fibre. Filament track lengths from 80 to $125 \mu \mathrm{m}$ were generated with 2 and $3 \mathrm{~mm}$ plate thickness, respectively. The surface aberration stretched the laser energy over a large depth of focus in a similar way to an axicon forming a Bessellike beam ${ }^{42}$. In this way, high pulse energy was applied without inducing distortion from Kerr lens focusing and plasma defocusing effects, while retaining the nonlinear interaction benefits of narrowing modification to a sub-diffractionlimited size.

The experimental approach was adapted from ref. ${ }^{45}$ wherein a standard singlemode telecommunication fibre (Corning, SMF-28) was mounted in contact with the bottom surface of the aberration plates (Fig. 1). Index matching oil (Cargille, 50350) was applied to fill the gap between the fibre and plate and remove cylindrical aberration by the fibre. Laser filaments were aligned laterally to $\pm 1 \mu \mathrm{m}$ precision to bisect the waveguide core (Fig. $3 \mathrm{f}$ ), and shifted vertically to illuminate any portion of core and cladding (Fig. $3 \mathrm{~g}$ ) by using a three-axis motion and alignment system (Aerotech Inc., Aerotech-PlanarDL-00 XY and ANT130-060-L $\mathrm{Z}$ ) having $3 \mathrm{~nm}$ resolution. Filament positions in the fibre cross-section (Fig. $3 \mathrm{f}, \mathrm{g}$ ) were verified by backlighting the fibre with an optical microscope

(Olympus, BX51).

The laser pulse energy was adjusted upward from the low-contrast conditions until the optical contrast of filament tracks forming in the fibre was observed to darken and indicate the onset of micro-explosion, opening hollow-filament shapes inside of the fibre. Single-pulse exposure of 3 and $7 \mu \mathrm{J}$ energy opened hollow filaments of $80 \mu \mathrm{m}$ to $125 \mu \mathrm{m}$ length, respectively, with silica plates of 2 and $3 \mathrm{~mm}$ thickness, respectively. The axial focusing was optimised to avoid the burning or evaporating of the RI-matching oil and minimise ablative machining and nearsurface damage at the fibre cladding surface. In this way, blind ( $2 \mathrm{~mm}$ plate) or fully open $(3 \mathrm{~mm}$ ) holes (Fig. $3 \mathrm{~g}$ ) were formed in the fibre core and cladding. The filament tracks could be aligned side-by-side to $\sim 1 \mu \mathrm{m}$ spacing and remain isolated without breaking through or undergoing beam propagation or processing distortion by the pre-existing track. Filament tracks were assembled into a tightly packed linear array and precisely centred along with the fibre core (Fig. 3f, g) for evaluation for FBG responses.

Nanohole morphology. The nano-scale formation of high-aspect ratio holes into the optical fibre was examined by mechanically cleaving and optically polishing a laser-exposed fibre at an oblique angle $\left(\sim 30^{\circ}\right)$ (Fig. 3a). A filament array of nanoholes was generated through the cladding and core waveguide regions on $1.072 \mu \mathrm{m}$ period, using $4.5 \mu \mathrm{J}$ pulse energy, and $2 \mathrm{~mm}$ thick aberration plate. The cleaved facet was polished with silicon carbide paper (1200 grit), whereas wetted with a few drops of distilled water. After cleaning with acetone and isopropanol, the polished fibre facet (Fig. 3a) was viewed in orthogonal alignment with an SEM (Hitachi, SU5000), providing high contrast views of the full fibre cross-section, shown in Fig. 3b-d. The SEM images confirmed a $\sim 200 \mathrm{~nm}$ diameter of the open nanoholes forming through a majority of the filament length, defining a highaspect ratio of $\sim 500$ times. Hole-to-hole breakthrough was not observed over most of the filament length for periods as small as $\Lambda=1.072 \mu \mathrm{m}$, or for identical nanohole arrays opened to the larger diameter (i.e., $350 \mathrm{~nm}$, Fig. 3e) after chemical etching.

Capillary flow. Optical microscopy was used for confirming and monitoring the wetting, capillary flow and evaporation of liquids with various values of RI (Table 1) into and out of the laser-formed nanoholes, providing optical images (Fig. 3g) and video recordings (Supplementary Movies $1 \& 2$ ). The wetting times varied from sub-seconds for solvents (i.e., water, methanol, acetone, etc.) (Merck) to tens of seconds for index matching oils (Cargile). Dichloromethane (DCM) and acetone were applied to remove oils and to clean nanoholes before immersing the fibre in a new liquid.

FBG characterisation. At low pulse energy exposure ( $350 \mathrm{~nJ})$, low-contrast filament arrays were previously shown to assemble in silica fibre on $\Lambda=0.536 \mu \mathrm{m}$ period and provide strong first-order Bragg grating responses at $1550 \mathrm{~nm}^{45}$. However, the opening of nanoholes with the higher pulse energy (3-7 $\mu \mathrm{J})$ required in the present work resulted in filament distortion and breakthrough of holes when positioned on a similar first-order period. The formation of isolated nanoholes was verified by SEM (Fig. $3 \mathrm{~d}$ ) with a doubling of the period to $\Lambda=1.072 \mu \mathrm{m}$, thus enabling a second-order FBG response at $1550 \mathrm{~nm}$ wavelength. $\pi$-shifted FBGs were fabricated for narrowing the spectral response of the device to below $200 \mathrm{pm}$ linewidth $(3 \mathrm{~dB})$. FBG spectral responses were recorded real-time during the laser fabrication and chemical etching, or during the filling or evaporation of various liquids (Table 1). Reflection spectra were excited with a $1530-1610 \mathrm{~nm}$ wavelength broadband source (Thorlabs, ASE-FL7002), and recorded through an optical fibre circulator (Thorlabs, 6015-3-FC) by a high-resolution optical spectrum analyser (Anritsu, MS9740B). The influence of laser exposure and the number of filaments were evaluated. Supplementary Fig. 3 shows the influence and limitation of increasing device length on the reflection peak and bandwidth. A 600 and 1200 element array of nanohole filaments were selected to study the FBG responses to solvents, chemical etching, and to compare uniform and $\pi$-shift gratings.

Chemical etching. Femtosecond laser irradiation followed by chemical etching $(\text { FLICE })^{56-58}$ was adopted here for opening the nanohole diameter. FLICE extended the diameter of the laser-formed nanoholes in a predictable and reproducible way. The laser-formed FBGs were immersed in acetone and followed with DCM to remove oils and debris. Fibres were submerged in 5\% dilute HF acid solution for up to 7 mins. Longer etching times were found to degrade the FBG spectrum. After etching, the fibre was promptly immersed in distilled water, then IPA and left to air dry. Identical FBGs were prepared with 2, 4, and 6 min of etching and then spectrally characterised underfilling with all solvent and oil types listed in Table 1. Assessment by a combination of SEM morphology (Fig. 3e), FBG spectral responses (Fig. $5 \mathrm{f}$ and Supplementary Fig. 5), and simulations (Supplementary Fig. 1) provided an estimated etching rate on the nanohole diameter beginning more slowly at $40 \mu \mathrm{m}$ per minute in the first 2 minutes and rising to a steady value of $100 \mathrm{~nm} /$ minute thereafter.

Optical modelling. Simulation of the 3D light intensity distribution (Fig. 2b, c) and the spectral reflections (Fig. 2a and Supplementary Fig. 1) expected from a nanohole array positioned in the core waveguide of SMF-28 fibre was provided by commercial software (Lumerical Inc.), based on finite difference time domain (FDTD: 3D Electromagnetic Simulator) and eigenmode expansion methods (EME; MODE: waveguide simulator), respectively.

For the FDTD simulation, intensity patterns (Fig. 2b, c) were provided for a second-order FBG of 100 nanoholes on $\Lambda=1.072 \mu \mathrm{m}$ pitch and having a nanohole diameter of $300 \mathrm{~nm}$. Core and cladding RI values were matched to SMF-28 fibre at $1550 \mathrm{~nm}$ wavelength. A perfect electrical conductor was imposed at the centre-fibre symmetry plane $(z=0$ in Fig. $2 \mathrm{~b}, \mathrm{c})$ with absorbing boundary conditions on the outside borders. A spatially uniform mesh with grid size of $\Delta x=\Delta y=\Delta z=48 \mathrm{~nm}$ was applied with time steps of $\Delta t=0.092 \mathrm{fs}$. Long programme running timelimited simulations to FBGs having a linear array of 100 filaments that spanned $\sim 100 \mu \mathrm{m}$ along with the fibre. Intensity patterns did not deviate materially when compared with the simulation of larger arrays of up to 600 nanoholes.

The EME simulation provided reflection spectra of FBGs (Fig. 2a, Fig. 4b-e, Supplementary Fig. 1, Supplementary Fig. 3, and Supplementary Fig. 6) containing arrays of 600 or 1200 nanoholes that were spaced uniformly or $\pi$-shifted respectively. A non-uniform spatial mesh was defined as having the finest pitch for lateral dimension beginning at $\Delta y=\Delta z=10 \mathrm{~nm}$ inside the fibre core array, and gradually increasing to $400 \mathrm{~nm}$ in the cladding. A uniformly small pitch of $\Delta x=6 \mathrm{~nm}$ was maintained axially. A unit cell was defined around the $\Lambda=1.072 \mu \mathrm{m}$ period of nanoholes and sandwiched by a pair of periodic boundary conditions. The programme provided a fundamental single-mode having a meanfield diameter of $\sim 10 \mu \mathrm{m}$ and returned reflection spectra through the S-parameter function.

\section{Data availability}

The data collected and generated during the study are available from the corresponding authors upon reasonable request.

\section{Code availability}

The codes used in the simulations are available from the corresponding authors upon reasonable request.

Received: 25 January 2021; Accepted: 15 October 2021; Published online: 03 November 2021

\section{References}

1. Kersey, A. D. et al. Fiber grating sensors. J. Lightwave Technol. 15, 1442-1463 (1997).

2. Hill, K. O. \& Meltz, G. 1997. Fiber Bragg grating technology fundamentals and overview. J. Lightwave Technol. 15, 1263-1276 (1997).

3. Liang, W., Huang, Y., Xu, Y., Lee, R. K. \& Yariv, A. 2005. Highly sensitive fiber Bragg grating refractive index sensors. Appl. Phys. Lett. 86, 151122 (2005). 
4. Guo, K., He, J. and Wang, Y. Fabrication of side-polished fiber Bragg grating for refractive index sensor. In Advanced Sensor Systems and Applications VIII (SPIE, 2018)

5. Li, B. et al. Ultra-abrupt tapered fiber Mach-Zehnder interferometer sensors. Sensors 11, 5729-5739 (2011).

6. Kakarantzas, G., Dimmick, T. E., Birks, T. A., Le Roux, R. \& Russell, P. S. J. Miniature all-fiber devices based on $\mathrm{CO} 2$ laser microstructuring of tapered fibers. Opt. Lett. 26, 1137-1139 (2001).

7. Fang, X., Liao, C. R. \& Wang, D. N. Femtosecond laser fabricated fiber Bragg grating in microfiber for refractive index sensing. Opt. Lett. 35, 1007-1009 (2010).

8. $\mathrm{Fu}, \mathrm{H}$. et al. Microchanneled chirped fiber Bragg grating formed by femtosecond laser-aided chemical etching for refractive index and temperature measurements. IEEE Photonics Technol. Lett. 20, 1609-1611 (2008).

9. Martelli, C. et al. Micromachining structured optical fibers using focused ion beam milling. Opt. Lett. 32, 1575-1577 (2007).

10. Debord, B., Amrani, F., Vincetti, L., Gérôme, F. \& Benabid, F. Hollow-core fiber technology: the rising of "gas photonics". Fibers 7, 16 (2019).

11. Jha, R., Villatoro, J., Badenes, G. \& Pruneri, V. Refractometry based on a photonic crystal fiber interferometer. Opt. Lett. 34, 617-619 (2009).

12. Lee, H. W. et al. Optofluidic refractive-index sensor in step-index fiber with parallel hollow micro-channel. Opt. Express 19, 8200-8207 (2011).

13. Li, X., Shao, Y., Yu, Y., Zhang, Y. \& Wei, S. A highly sensitive fiber-optic Fabry-Perot interferometer based on internal reflection mirrors for refractive index measurement. Sensors 16, 794 (2016).

14. Cusano, A., Paladino, D. \& Iadicicco, A. Microstructured fiber Bragg gratings. J. Lightwave Technol. 27, 1663-1697 (2009).

15. Grillet, C. et al. Compact tunable microfluidic interferometer. Opt. Express 12, 5440-5447 (2004).

16. Psaltis, D., Quake, S. R. \& Yang, C. Developing optofluidic technology through the fusion of microfluidics and optics. Nature 442, 381-386 (2006).

17. Monat, C., Domachuk, P. \& Eggleton, B. J. Integrated optofluidics: a new river of light. Nat. Photonics 1, 106-114 (2007).

18. Chiavaioli, F., Gouveia, C. A., Jorge, P. A. \& Baldini, F. Towards a uniform metrological assessment of grating-based optical fiber sensors: from refractometers to biosensors. Biosensors 7, 23 (2017).

19. Yin, M. J. et al. Recent development of fiber-optic chemical sensors and biosensors: mechanisms, materials, micro/nano-fabrications and applications. Coord. Chem. Rev. 376, 348-392 (2018).

20. Correia, R., James, S., Lee, S. W., Morgan, S. P. \& Korposh, S. Biomedical application of optical fibre sensors. J. Opt. 20, 073003 (2018)

21. Sun, D., Ran, Y. \& Wang, G. Label-free detection of cancer biomarkers using an in-line taper fiber-optic interferometer and a fiber Bragg grating. Sensors 17, 2559 (2017).

22. Dragomir, A., Nikogosyan, D. N., Zagorulko, K. A., Kryukov, P. G. \& Dianov, E. M. Inscription of fiber Bragg gratings by ultraviolet femtosecond radiation. Opt. Lett. 28, 2171-2173 (2003).

23. Pitruzzello, G. \& Krauss, T. F. Photonic crystal resonances for sensing and imaging. J. Opt. 20, 073004 (2018).

24. Yang, D. Q. et al. Photonic crystal nanobeam cavities for nanoscale optical sensing: a review. Micromachines 11, 72 (2020).

25. Fernández Gavela, A., Grajales García, D., Ramirez, J. C. \& Lechuga, L. M. Last advances in silicon-based optical biosensors. Sensors 16, 285 (2016).

26. Scullion, M. G., Arita, Y., Krauss, T. F. \& Dholakia, K. Enhancement of optical forces using slow light in a photonic crystal waveguide. Optica 2, 816-821 (2015).

27. Luan, E., Shoman, H., Ratner, D. M., Cheung, K. C. \& Chrostowski, L. Silicon photonic biosensors using label-free detection. Sensors 18, 3519 (2018).

28. Zhi, Y., Yu, X. C., Gong, Q., Yang, L. \& Xiao, Y. F. Single nanoparticle detection using optical microcavities. Adv. Mater. 29, 1604920 (2017).

29. Xu, Y., Lu, P., Chen, L. \& Bao, X. Recent developments in micro-structured fiber optic sensors. Fibers 5, 3 (2017).

30. Urrutia, A., Del Villar, I., Zubiate, P. \& Zamarreño, C. R. A comprehensive review of optical fiber refractometers: Toward a standard comparative criterion. Laser Photonics Rev. 13, 1900094 (2019).

31. Liu, Z., Zhang, Z. F., Tam, H. Y. \& Tao, X. Multifunctional smart optical fibers: materials, fabrication, and sensing applications. Photonics 6, 48 (2019).

32. Liu, Y. et al. Compact microfiber Bragg gratings with high-index contrast. Opt. Lett. 36, 3115-3117 (2011).

33. Nayak, K. P. et al. Cavity formation on an optical nanofiber using focused ion beam milling technique. Opt. Express 19, 14040-14050 (2011).

34. Zhao, P., Li, Y., Zhang, J., Shi, L. \& Zhang, X. Nanohole induced microfiber Bragg gratings. Opt. Express 20, 28625-28630 (2012).

35. Li, W., Du, J., Truong, V. G. \& Nic Chormaic, S. Optical nanofiber-based cavity induced by periodic air-nanohole arrays. Appl. Phys. Lett. 110, 253102 (2017).
36. Nayak, K. P. \& Hakuta, K. Photonic crystal formation on optical nanofibers using femtosecond laser ablation technique. Opt. Express 21, 2480-2490 (2013).

37. Yang, R., Yu, Y. S., Chen, C., Chen, Q. D. \& Sun, H. B. Rapid fabrication of microhole array structured optical fibers. Opt. Lett. 36, 3879-3881 (2011).

38. Bhuyan, M. K. et al. High aspect ratio nanochannel machining using single shot femtosecond Bessel beams. Appl. Phys. Lett. 97, 081102 (2010).

39. Bhuyan, M. K. et al. High aspect ratio taper-free microchannel fabrication using femtosecond Bessel beams. Opt. Express 18, 566-574 (2010).

40. Courvoisier, F. et al. Surface nanoprocessing with nondiffracting femtosecond Bessel beams. Opt. Lett. 34, 3163-3165 (2009).

41. Hof, L. A. \& Abou Ziki, J. Micro-hole drilling on glass substrates-a review. Micromachines 8, 53 (2017).

42. Chen, T. et al. Reconstructing of embedded high-aspect-ratio nano-voids generated by ultrafast laser bessel beams. Micromachines 11, 671 (2020).

43. Rapp, L. et al. High aspect ratio micro-explosions in the bulk of sapphire generated by femtosecond Bessel beams. Sci. Rep. 6, 1-6 (2016).

44. Alimohammadian, E., Ertorer, E., Uzeda, E. M., Li, J. \& Herman, P. R. Inhibition and enhancement of linear and nonlinear optical effects by conical phase front shaping for femtosecond laser material processing. Sci. Rep. 10, $1-13(2020)$

45. Ertorer, E., Haque, M., Li, J. \& Herman, P. R. Femtosecond laser filaments for rapid and flexible writing of fiber Bragg grating. Opt. Express 26, 9323-9331 (2018).

46. Rahnama, A., Mahmoud Aghdami, K., Kim, Y. H. \& Herman, P. R. Ultracompact lens-less "Spectrometer in Fiber" based on chirped filamentarray gratings. Adv. Photonics Res. 1, 2000026 (2020).

47. Rahnama, A., Kim, Y. H., Aghdami, K. M. and Herman, P. R. Visible-light, All-fiber Spectrometer Based on Radiative Emission From a Chirped Filament Grating Array. In Specialty Optical Fibers Confernce, SoTh1H-4 (Optical Society of America, 2020)

48. Herman, P. R. et al. Femtosecond Laser Nano-Filament Explosion: Opening Fiber Bragg Gratings for Opto-Fluidic Sensing. In Conference on Lasers and Electro-Optics (CLEO), STh1H.2 (Optical Society of America, 2020).

49. Zhang, G. et al. Ultrashort Bessel beam photoinscription of Bragg grating waveguides and their application as temperature sensors. Photonics Res. 7 , 806-814 (2019).

50. Bernier, M., Trépanier, F., Carrier, J. \& Vallée, R. High mechanical strength fiber Bragg gratings made with infrared femtosecond pulses and a phase mask. Opt. Lett. 39, 3646-3649 (2014).

51. Gu, X., Guan, L., He, Y., Zhang, H. B. \& Herman, R. High-strength fiber Bragg gratings for a temperature-sensing array. IEEE Sens. J. 6, 668-671 (2006).

52. Zhou, K. et al. A refractometer based on a micro-slot in a fiber Bragg grating formed by chemically assisted femtosecond laser processing. Opt. Express $\mathbf{1 5}$ 15848-15853 (2007)

53. Rahnama, A., Dadalyan, T., Mahmoud Aghdami, K., Galstian, T. and Herman, P. R. In-fiber switchable polarization filter based on liquid crystal filled hollow-filament bragg gratings. Adv. Opt. Mater. 9, 2100054 (2021).

54. Rahnama, A., Kim, Y., Aghdami, K. M. and Herman, P. R., 2021, March. 2D filament grating array: enabling an efficient, high-resolution lens-less all-fiber spectrometer. In Frontiers in Ultrafast Optics: Biomedical, Scientific, and Industrial Applications XXI, 116760Y (International Society for Optics and Photonics, 2021).

55. Saunders, J. E., Sanders, C., Chen, H. \& Loock, H. P. Refractive indices of common solvents and solutions at $1550 \mathrm{~nm}$. Appl. Opt. 55, 947-953 (2016).

56. Haque, M. \& Herman, P. R. Chemical-assisted femtosecond laser writing of optical resonator arrays. Laser Photonics Rev. 9, 656-665 (2015).

57. Djogo, G. et al. Femtosecond laser additive and subtractive micro-processing: enabling a high-channel-density silica interposer for multicore fibre to siliconphotonic packaging. Int. J. Extrem. Manuf. 1, 045002 (2019).

58. Bellouard, Y. et al. Stress-state manipulation in fused silica via femtosecond laser irradiation. Optica 3, 1285-1293 (2016).

\section{Acknowledgements}

The authors are grateful to Gligor Djogo and Dr. Stephen Ho for guidance on chemica etching and SEM imaging, to Dr. Jianzhao Li for experimental support and to Dr. Ehsan Alimohammadian for aberration tuning of filament shape. The authors are also appreciative of professor Xijia Gu, Michael Bakaic, and Nicholas Burgwin for providing fibre proof tester and their advice on fibre tensile strength measurements. This work was supported by the Natural Sciences and Engineering Research Council of Canada (NSERC, grant nos.: STPGP 521526-18, CREAT 484907-16), the Edward S. Rogers Sr. Graduate Scholarship, and Mitacs Accelerate Fellowship (IT16189). 


\section{Author contributions}

Device fabrication and characterisation were conducted by A.R. and K.M.A. E.E. provided guidance with generating laser filaments and with SEM characterisation. K.M.A carried out the theoretical modelling. The manuscript was written by A.R., K.M.A, and P.R.H. and all authors contributed to editing. P.R.H. directed the overall project.

\section{Competing interests}

The authors declare no competing interests.

\section{Additional information}

Supplementary information The online version contains supplementary material available at https://doi.org/10.1038/s41467-021-26671-4.

Correspondence and requests for materials should be addressed to Keivan Mahmoud Aghdami or Abdullah Rahnama.

Peer review information Nature Communications thanks the anonymous reviewer(s) for their contribution to the peer review of this work. Peer reviewer reports are available.
Reprints and permission information is available at http://www.nature.com/reprints

Publisher's note Springer Nature remains neutral with regard to jurisdictional claims in published maps and institutional affiliations.

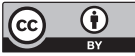

Open Access This article is licensed under a Creative Commons Attribution 4.0 International License, which permits use, sharing, adaptation, distribution and reproduction in any medium or format, as long as you give appropriate credit to the original author(s) and the source, provide a link to the Creative Commons license, and indicate if changes were made. The images or other third party material in this article are included in the article's Creative Commons license, unless indicated otherwise in a credit line to the material. If material is not included in the article's Creative Commons license and your intended use is not permitted by statutory regulation or exceeds the permitted use, you will need to obtain permission directly from the copyright holder. To view a copy of this license, visit http://creativecommons.org/licenses/by/4.0/.

(C) The Author(s) 2021 